\title{
Obligate anaerobic Salmonella typhimurium strain YB1 treatment on xenograft tumor in immunocompetent mouse model
}

\author{
BIN YU ${ }^{1,2}$, LEI SHI ${ }^{2}$, BAO-ZHONG ZHANG ${ }^{2}$, KE ZHANG $^{3}$, XIAO PENG $^{1}$, HAN-BEN NIU $^{1}$ and JUN-LE QU ${ }^{1}$ \\ ${ }^{1}$ Key Laboratory of Optoelectronic Devices and Systems of Ministry of Education and Guangdong Province, \\ College of Optoelectronic Engineering, Shenzhen University, Shenzhen, Guangdong 518060; \\ ${ }^{2}$ Department of Biochemistry, LKS Faculty of Medicine; ${ }^{3}$ Department of Microbiology, \\ The University of Hong Kong, Pokfulam, Hong Kong, SAR, P.R. China
}

Received July 28, 2014; Accepted March 27, 2015

DOI: $10.3892 / 01.2015 .3302$

\begin{abstract}
The present authors have previously reported a novel approach to genetically engineer Salmonella typhimurium for the medically important therapeutic strategy of using bacterial agents to target malignant tumors in a breast cancer tumor-bearing nude mouse model. However, studying an immunocompromised mouse model for cancer therapy is insufficient, as certain crucial information about the influence of the immune system may be missing. In the present study, inoculation of the Salmonella strain, YB1, into a colon cancer tumor-bearing immunocompetent mouse model was investigated. The present study determined the tumor targeting efficiency, antitumor potential, the effects of multiple treatments and the systemic toxicity. Intravenous inoculation of YB1 in BALB/c mice exhibited high antitumor effects and also greatly increased the tumor targeting ability and safety compared with the previously-reported nude mouse model. In addition, repeated administration of YB1 further enhanced this effect. Furthermore, no marked toxicity was observed with YB1 treatment, while the VNP20009 and SL7207 strains demonstrated certain adverse effects. The findings of the present study indicate that the YB1 strain is effective and safe in targeting a colon cancer tumor in an immunocompetent mouse model.
\end{abstract}

Correspondence to: Dr Bin Yu or Professor Jun-Le Qu, Key Laboratory of Optoelectronic Devices and Systems of Ministry of Education and Guangdong Province, College of Optoelectronic Engineering, Shenzhen University, 3688 Nanhai Road, Shenzhen, Guangdong 518060, P.R. China

E-mail: yubin1982@msn.com

E-mail: jlqu70@gmail.com

Key words: Salmonella, YB1 strain, synthetic biology, bacterial cancer therapy

\section{Introduction}

The potential use of certain obligate and facultative anaerobic bacteria in cancer treatment has been investigated for numerous years (1). Bacteria, including Bifidobacterium, Clostridium and Salmonella, have been demonstrated to preferentially target and replicate in the hypoxic and necrotic regions of tumors, resulting in tumor repression (2-9). In our previous study, in order to strengthen the tumor hypoxia targeting efficiency and antitumor effect, a synthetic biology approach was used to generate a novel Salmonella strain, YB1 (10). The principle of YB1 is to regulate an essential gene (asd) of Salmonella using a hypoxia-induced promoter (10). Since Salmonella is sensitive to the expression of the asd gene, any leaky expression will destroy the entire regulation. Therefore, a genetic circuit was designed to neutralize the leakage, which was controlled by an anaerobic promoter in order to regulate forward transcription of $a s d$, and another aerobic promoter was constructed to generate anti-sense asd mRNA (10). With this combination, YB1 only survived in an atmosphere with $<0.5 \% \mathrm{O}_{2}$, which is equivalent to the anaerobic conditions inside solid tumor tissue (11), and the growth of YB1 was inhibited when exposed to increased oxygen levels that are equivalent to those in a normal organ $\left(2-9 \% \mathrm{O}_{2}\right)$ without additional diaminopimelic acid (DAP) $(10,12)$. In the breast tumor-bearing nude mouse model, YB1 specifically colonized and proliferated in hypoxic/necrotic areas of the tumor, and retarded tumor growth (10).

In the present study, the antitumor effects of the YB1 strain were further investigated. The tumor targeting efficiency and safety evaluation were investigated in a solid tumor model using the colon cancer cell line, CT26. In addition, inoculation of YB1 in an immunocompetent mouse model (BALB/c mice) was compared with the previously-reported immunocompromised mouse model (nude mice). Furthermore, multiple YB1 injection treatments were assessed and the treatment of different tumor sizes was investigated. The present study, using the genetically engineered YB1 strain, provides preclinical data on the efficiency and safety of Salmonella mediated cancer therapy, which may further facilitate its optimization to improve the anticancer effect and reduce adverse effects in future clinical trials. 
$\bar{A}$

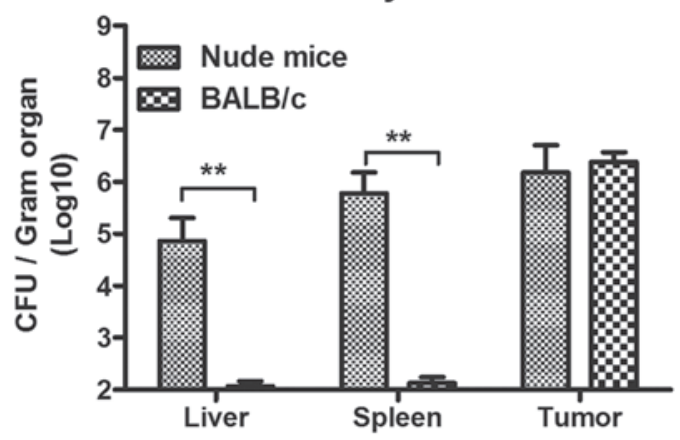

C

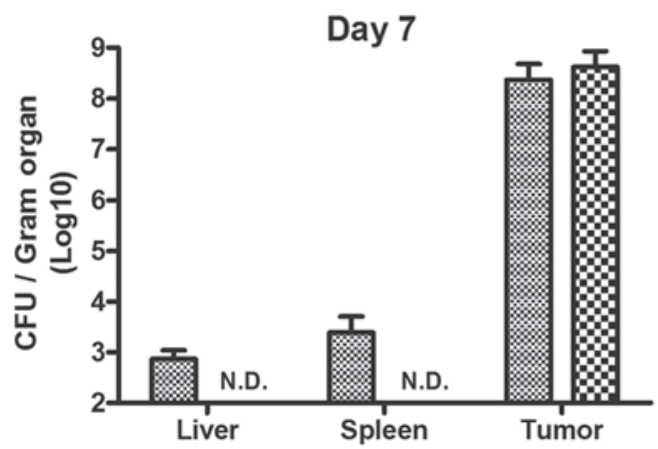

B

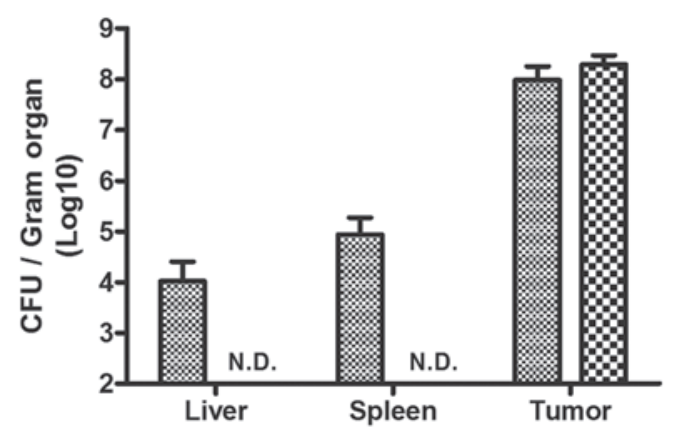

D

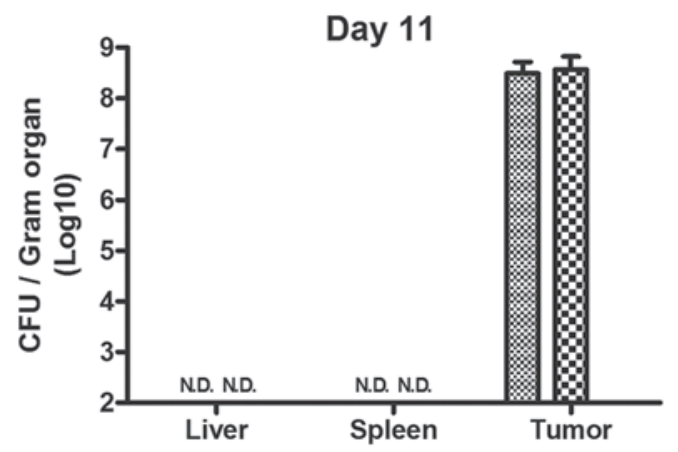

Figure 1. CFU test of YB1 in colon tumor-bearing nude and BALB/c mice. Nude and BALB/c mice with CT26 tumor received tail vein intravenous injections of YB1. Mice were euthanized at various time points, including days (A) 1, (B) 3, (C) 7 and (D) 11. The liver, spleen and tumor were collected and homogenized. CFU counts per gram of tissue are presented as the mean \pm standard deviation $\left(\mathrm{n}=6\right.$ mice). ${ }^{* *} \mathrm{P}<0.01$. CFU, colony-forming unit; N.D., not detected.

\section{Materials and methods}

Bacteria, cell lines and animals. The Salmonella typhimurium strain, SL7207, was obtained from lab stock (13). The VNP20009 strain was purchased from the American Type Culture Collection (\#202165; Manassas, VA, USA). The YB1 strain was constructed as described previously (10). Bacteria were cultured in lysogeny broth (LB) medium (Sigma-Aldrich, St. Louis, MO, USA) supplemented with chloramphenicol and DAP (Sigma-Aldrich) at $37^{\circ} \mathrm{C}$. The CT26 colon carcinoma cell line was provided by Dr Songyue Zheng (University of Hong Kong, Hong Kong, China). Cells were cultured in high-glucose Dulbecco's modified Eagle's medium containing $10 \%$ fetal bovine serum and $1 \%$ penicillin-streptomycin (Gibco Life Technologies, Carlsbad, CA, USA). Four-week-old female BALB/c nude mice (weight range, 16-20 g) and eight-week-old BALB/c mice (weight range, 18-20 g) were purchased from the Laboratory Animal Unit of The University of Hong Kong. The research protocols were approved and followed by the committee on the Use of Live Animals in Teaching and Research of the University of Hong Kong (CULATR no. 2689-12). Antibiotic and chemical working solutions were prepared as follows: Chloramphenicol, $25 \mu \mathrm{g} / \mathrm{ml}$ in methanol; DAP, $50 \mu \mathrm{g} / \mathrm{ml}$ in water.

Tumor-bearing mouse model. A total $5 \times 10^{5}$ CT26 cells were injected into the fat pad of the chest in nude mice or BALB/c mice. The tumor volumes were calculated using the following formula: $4 / 3 \pi$ (height $\mathrm{x}$ width $\left.{ }^{2}\right) / 8$. When the tumors grew to $\sim 500$ or $100 \mathrm{~mm}^{3}$, the mice underwent bacterial treatment and were grouped as follows: YB1-treated BALB/c group; YB1-1-treated nude group; VNP20009-treated BALB/c group; and SL7207-treated BALB/c group. Phosphate-buffered saline (PBS) treatment was used as a control. If the tumor size reached $20 \mathrm{~mm}$ in height or the volume was $\geq 4,000 \mathrm{~mm}^{3}$, the mouse was euthanized (14). To measure YB1 distribution following treatment at different time points in nude mice and BALB/c mice, $5 \times 10^{7}$ colony-forming units (CFU) of YB1, VNP20009 or SL7207 were injected intravenously into CT26 tumor-bearing mice through the tail vein. The mice were sacrificed by intraperitoneal injection with pentobarbitone (University of Hong Kong) at different time points (day 1,3,7 or 11). The calculation of YB1 in tissues, survival rates and body weight analysis followed the methods previously described (10).

Tumor targeting potential and clearance of YB1. The tumor targeting potential and the clearance of YB1 in normal tissues were compared in immunocompetent (nude) and immunocompromised (BALB/c) mice. CT26 tumor cells were injected subcutaneously into nude and BALB/c mice. When the tumor volumes reached $\sim 500 \mathrm{~mm}^{3}$, a single shot of YB1 was injected intravenously through the tail vein. At the indicated time points (day 1, 3, 7 or 11), the mice were euthanized by intraperitoneal injection with pentobarbitone and the liver, spleen and tumor were isolated, homogenized in LB and cultured on LB agar plates supplemented with antibiotics and DAP.

Multiple-shot YB1 treatment in BALB/c mice CT26 cancer model. A total of $1 \times 10^{5} \mathrm{CT} 26$ tumor cells were injected subcu- 

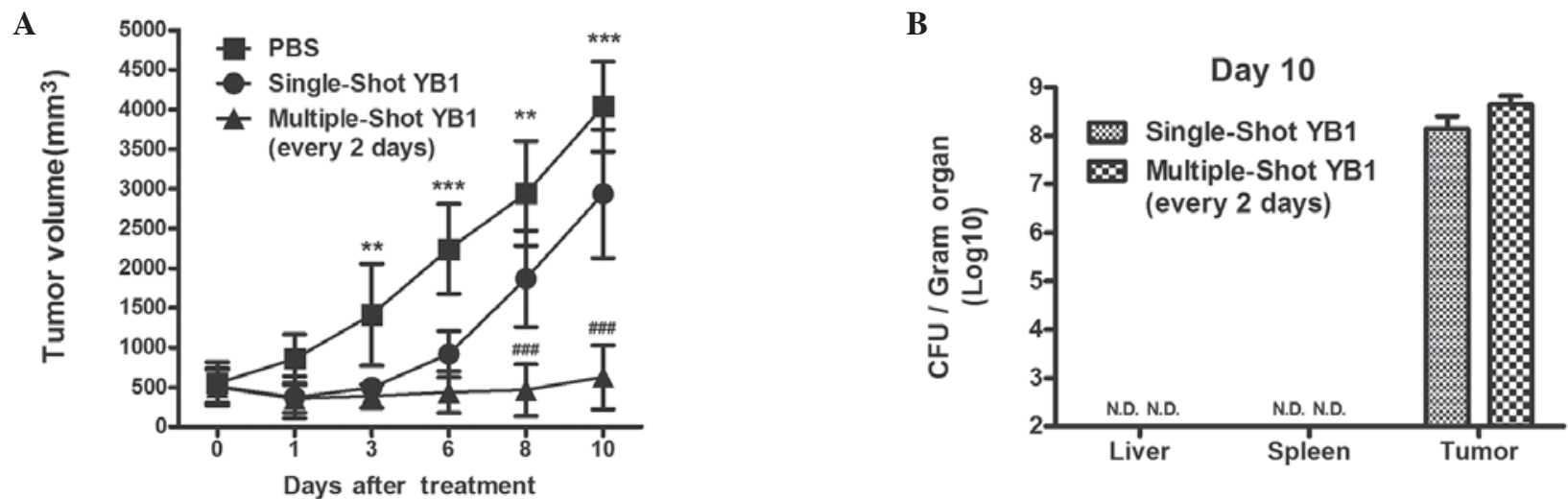

Figure 2. Repression of tumor growth by YB1 treatment. (A) When the tumor volume of CT26 tumor-bearing BALB/c mice reached 500 mm ${ }^{3}$, YB1 was injected once (single-shot group) or every 2 days for total of 4 times (multiple-shot group; $\mathrm{n}=5$, mean \pm standard deviation). (B) $\mathrm{CFU}$ counts indicated YB1 distribution in organs after 10 days of treatment. ${ }^{* *} \mathrm{P}<0.01$ and ${ }^{* * *} \mathrm{P}<0.001$, PBS group vs. YB1 groups; ${ }^{\# \#} \mathrm{P}<0.001, \mathrm{YB} 1$ single-shot vs. multiple-shot groups. PBS, phosphate-buffered saline; CFU, colony-forming unit.

taneously into $10 \mathrm{BALB} / \mathrm{c}$ mice. When the tumor volumes reached $\sim 500 \mathrm{~mm}^{3}$, mice in the 'single-shot YB1' group $(\mathrm{n}=5)$ were intravenously injected with a single-shot of YB1, whereas mice in the 'multiple-shot YB1' group $(n=5)$ received an intravenous injection of YB1 every two days (days 0, 2, 4 and 6). The tumor sizes in the two groups were measured on days 0 , $1,3,6,8$ and 10 . On day 10 , all mice were sacrificed by intraperitoneal injection with pentobarbitone. The liver, spleen and tumor were then isolated, homogenized in LB and cultured on LB agar plates supplemented with DAP to determine YB1 distribution.

Single-shot of different Salmonella strains in small CT26 tumor models. A total of $1 \times 10^{5} \mathrm{CT} 26$ tumor cells were injected subcutaneously into $20 \mathrm{BALB} / \mathrm{c}$ and 10 nude mice. When the tumor volumes reached $100 \mathrm{~mm}^{3}$, a single-shot of YB1 $(\mathrm{n}=5)$, VNP20009 (n=5), SL7207 (n=5), or PBS (n=5) was injected intravenously into BALB/c mice, whereas nude mice were intravenously injected with a single-shot of YB1 $(n=5)$ or PBS $(n=5)$. The tumor sizes in all groups were measured on days $0,1,3,6,8,10$ and 12 . On day 12 , all mice were sacrificed by intraperitoneal injection with pentobarbitone. The liver, spleen and tumor were then isolated, homogenized in LB and cultured on LB agar plates supplemented with DAP to determine the distribution of YB1, VNP20009 and SL7207.

Statistical analysis. Student's t-test was used to analyze the significance of the results of the CFU test and tumor growth measurements. Analyses were performed using GraphPad Prism 5 (GraphPad Software, Inc., La Jolla, CA, USA). Data are expressed as the mean \pm standard deviation and $\mathrm{P}<0.01$ was considered to indicate a statistically significant difference.

\section{Results}

Accumulation of YB1 in CT26 tumors and normal tissues in nude and $B A L B / c$ mice. For the nude mice group, the results were similar to those observed in our previous study in a breast cancer tumor-bearing nude mouse model (10). Between $1 \times 10^{5}$ and $1 \times 10^{6} \mathrm{CFU} / \mathrm{g}$ of bacteria were observed in all the tested tissues on day 1 (Fig. 1A). After one day, the YB1 levels in the liver and spleen declined rapidly. In the tumor tissues, the YB1 levels increased until they reached a plateau of $1 \times 10^{8} \mathrm{CFU} / \mathrm{g}$ at day 3 , which remained stable until day 11 (Fig. 1B-D). The tumor to liver ratio was 9,000:1 on day 3 (Fig. 1B; $\mathrm{P}<0.05$ ) and 300,000:1 on day 7 (Fig. 1C; $\mathrm{P}<0.05$ ). By day 11, YB1 was completely eliminated from the liver and spleen (Fig. 1D, P<0.01). Notably, the YB1 levels reduced much more quickly in the BALB/c mice compared with the nude mice. Within $24 \mathrm{~h}$ after treatment with YB1, the YB1 levels reduced to $\sim 1 \times 10^{2} \mathrm{CFU} / \mathrm{g}$ in the liver and spleen (Fig. 1A), while between days 3 and 11 no YB1 was detected in the normal tissues (Fig. 1B-D). YB1 levels in the tumor demonstrated a similar pattern in nude mice, which reached a plateau of $2-4 \times 10^{8} \mathrm{CFU} / \mathrm{g}$ at day 3 that was maintained until day 11 (Fig. 1B-D).

Antitumor effect of multiple-shot YB1 treatment in BALB/c mice CT26 cancer model (tumor size $\sim 500 \mathrm{~mm}^{3}$ ). Since a large amount of YB1 accumulated in the tumor, its antitumor effect was measured. Tumor growth (from a volume of $\sim 500 \mathrm{~mm}^{3}$ at the time of bacterial inoculation) following a single-shot YB1 treatment in BALB/c mice was initially inhibited for 3 days and then delayed compared with the PBS-treated group from days 3-10 ( $\mathrm{P}<0.01$ on days 3 and $8 ; \mathrm{P}<0.001$ on days 6 and 10) (Fig. 2A). Since YB1 was cleared in normal tissues within one day (Fig. 1), YB1 treatment was increased in tumor-bearing BALB/c mice via intravenous injections every two days (on days 2, 4, 6 and 8). The tumor growth was significantly repressed $(\mathrm{P}<0.001$ on days 8 and 10) and the volume of the tumors remained $\sim 500 \mathrm{~mm}^{3}$ in the 10 subsequent days (Fig. 2A). On day 10 following YB1 treatment, the single-shot and multiple-shot groups of mice were euthanized by intraperitoneal injection with pentobarbitone, and the bacterial number in different organs was counted. The residual YB1 in the liver and spleen of the two groups was eliminated. In the tumor tissues, the YB1 levels in the multiple-shot group were increased compared with the single-shot group $(\mathrm{P}<0.05$; Fig. $2 \mathrm{~B})$.

Antitumor effect with single-shot of different Salmonella strains in small CT26 tumor models $\left(\sim 100 \mathrm{~mm}^{3}\right)$. To determine the antitumor ability of YB1 in small tumors $\left(\sim 100 \mathrm{~mm}^{3}\right)$, a single-shot of YB1 was administrated to 
A

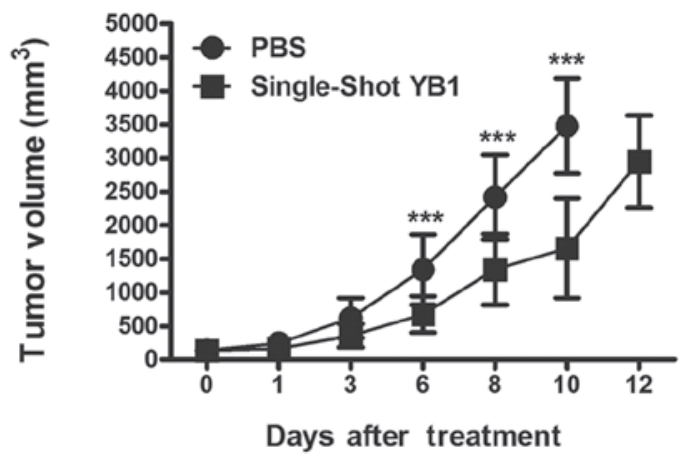

B

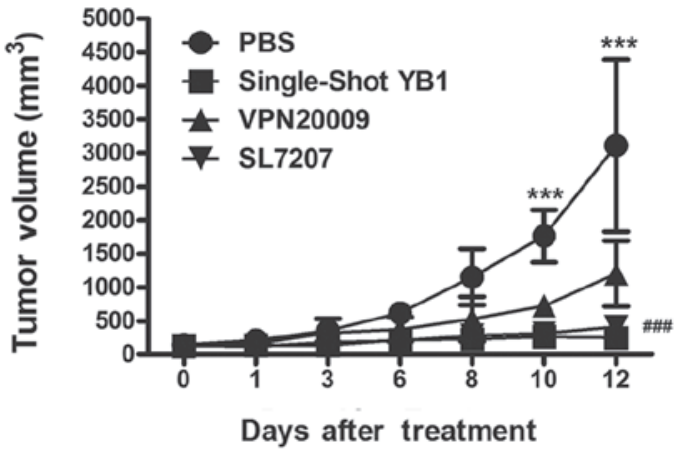

C

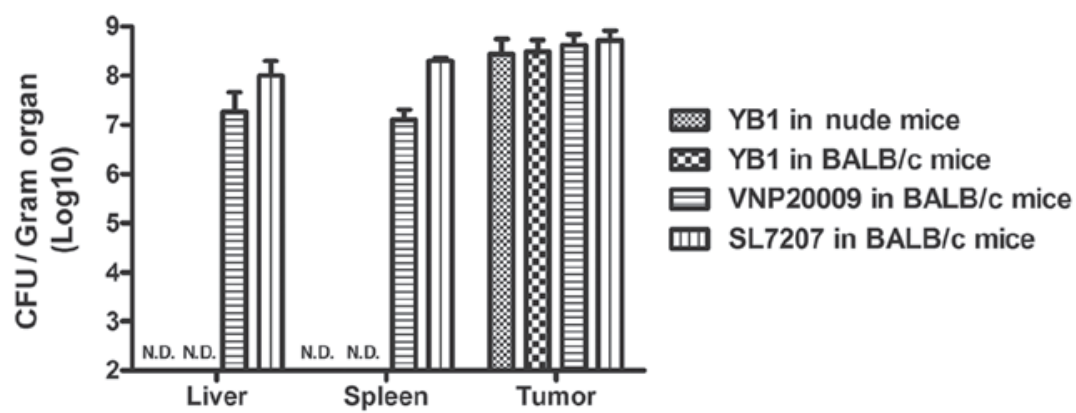

Figure 3. Repression of colon tumor growth by Salmonella strains in mice with a small tumor. CT26 colon tumor initial size $\sim 100$ mm ${ }^{3}$ in (A) nude mice or (B) BALB/c mice. Nude mice were treated with single-shot YB1 ( $\mathrm{n}=5$, mean \pm standard deviation). BALB/c mice were injected with PBS, YB1, VNP20009 or SL7207 ( $n=5$ each; mean \pm standard deviation). (C) On day 12 after treatment, the mice of each group were euthanized and the bacteria in the organs were

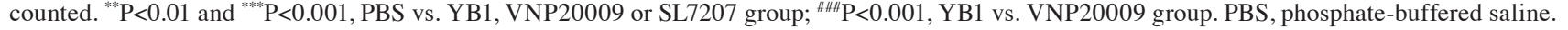

A

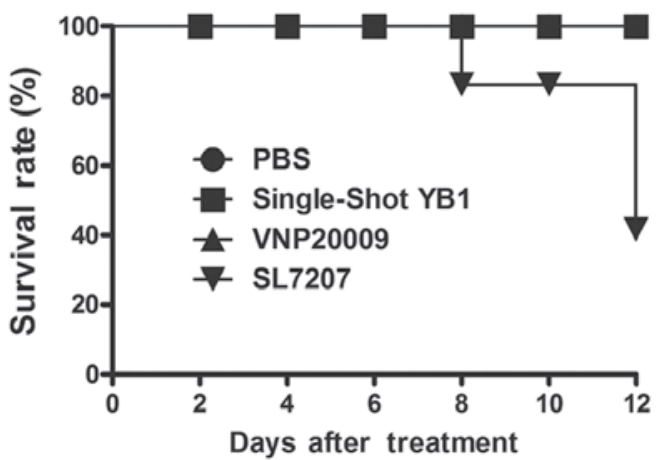

B

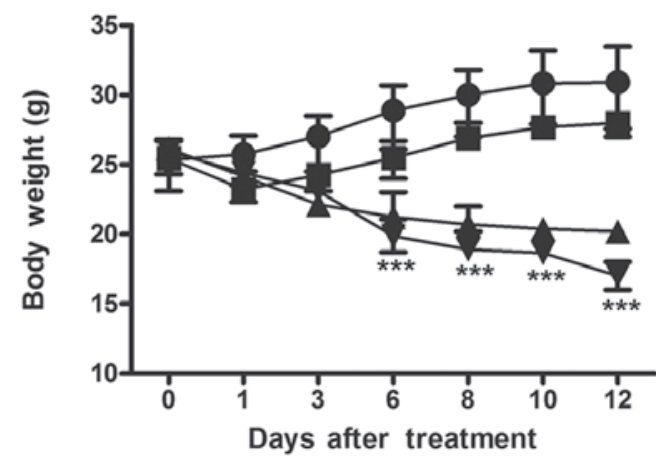

Figure 4. Evaluation of adverse effects of bacterial infection in CT26 colon tumor bearing BALB/c mice treated with PBS, YB1, VNP20009 or SL7207. (A) Survival rates and (B) body weights ( $\mathrm{n}=5$ each; mean \pm standard deviation) of the mice. ${ }^{* * *} \mathrm{P}<0.001$, PBS or YB1 group vs. VNP20009 or SL7207 treatment. PBS, phosphate buffered saline.

CT26 tumor-bearing nude and BALB/c mice (Fig. 3A and B). The results demonstrated that YB1 accumulated at approximately the same level in the two mouse models (Fig. 3C). The growth of the small tumors following inoculation with YB1 varied markedly, and tumor growth was delayed in nude mice but not in BALB/c mice (Fig. 3A). However, the overall growth of the tumor in $\mathrm{BALB} / \mathrm{c}$ mice treated with a single-shot YB1 was significantly reduced compared with the PBS-treated mice, within the 12-day observation period (Fig. 3B).

The VNP20009 treatment repressed tumor growth in BALB/c mice for 6 days; however the tumor continued to grow after day 6 (Fig. 3B). The SL7207-treated mice demonstrated approximately the same pattern in tumor growth repression as YB1 inoculation in BALB/c mice (Fig. 3B).
However, although YB1, VNP20009 and SL7207 accumulated at similar levels in the tumor $\left(2 \times 10^{8} \mathrm{CFU} / \mathrm{g}\right)$, significant differences were observed in the normal tissues (Fig. 3C). SL7207-inoculated mice demonstrated an uncontrolled infection as the bacterial level in the liver and spleen increased to $1 \times 10^{8} \mathrm{CFU} / \mathrm{g}$ (Fig. 3C). In VNP20009-treated mice, the bacterial levels were slightly reduced compared with the mice treated with SL7207, but were still $\sim 1 \times 10^{7} \mathrm{CFU} / \mathrm{g}$ in the liver and spleen (Fig. 3C).

Evaluation of the adverse effects of bacterial infection on weight gain and survival rate. Body weight and survival rate are major evaluation makers of treatment toxicity analysis for preclinical studies (15). Therefore, the adverse effects of the bacterial infection strategies used in the present study were 
assessed based on the survival rate and body weight. Mice with small CT26 tumor models $\left(\sim 100 \mathrm{~mm}^{3}\right)$ that were treated with single-shot YB1, VNP20009 or PBS survived during the 12-day observation period. However, a number of SL7207-treated mice succumbed on day 8 , and the overall survival rate within the 12-day observation period was $40 \%$ (Fig. 4A). Following the first day of inoculation, all the treatment groups demonstrated significant body weight loss compared with the PBS-treated group (YB1, P<0.01; VNP20009 and SL7207, P<0.05); however, only the YB1-treated group started to recover after day 1 $(\mathrm{P}<0.01)$, whereas the other groups demonstrated continuous weight loss throughout the 12 days of observation (VNP20009, SL7207, $\mathrm{P}<0.001$ on days 6-12). Although no mice succumbed in the VNP20009-treated group, their health condition was poorer than the YB1 group, due to the reduction in body weight following treatment (Fig. 4B).

\section{Discussion}

The physiology of solid tumors is different compared with normal tissues in certain aspects (16-20). Compared with normal organs, solid tumors consume a larger amount of oxygen and nutrients, a greater number of blood vessels supply the tissue and the vasculature within the tumor is highly abnormal with leaky vessel walls and reduced flow (16-18). Although tumors continuously generate new blood vessels, large regions of hypoxia are frequently observed in solid tumors $(11,21,22)$. These regions of hypoxia lead to problems in cancer treatment and can result in resistance to radiotherapy or chemotherapy (21). However, the microenvironment of the solid tumor provides a haven for certain obligate or facultative anaerobic bacteria, such as Salmonella $(23,24)$. When attenuated Salmonella strains are used as an anticancer vector, they are preferentially colonized in tumors with the ratio of titer being 1,000-10,000:1 in tumor over normal tissues. They may also evade monitoring by the immune system, and thus replicate and accumulate in the hypoxia region for long periods of time $(1,7,8,25)$. Although Salmonella preferentially accumulates in tumor tissues, a proportion colonizes the normal tissues $(10,26)$. In our previous study, recombineering technology was used to convert a Salmonella typhimurium strain into an 'obligate' anaerobe without otherwise interfering with the function of the bacterium. This avoids the problem of infection in normal aerobic tissues, as the modified strain lyses under these conditions. When in the hypoxic regions of a tumor, the bacterium thrives and functions as the wild type form as it is not compromised by an attenuation process. The efficacy of this approach was demonstrated by the effective tumor targeting and regression without damage to the normal organs in a situation where the unmodified parent strain is lethal to the host (10).

In our previous study (10), the animal model used to investigate Salmonella treatment included nude mice, which are deficient in T cell function. Mutations in the Foxnl gene in nude mice results in thymic aplasia and a lack of T cells, although the B cells remain unchanged (27). The immune system may aid bacteria to prevent cancer progression (28). A major obstacle for cancer immunotherapy is the ability of tumors to generate a microenvironment to evade the monitoring of the immune system. Although numerous methods have been developed to induce cancer immunity and certain interventions have demonstrated remarkably potent antitumor specific cytotoxic responses (29-32), the majority of tumors did not regress following these treatments and continued to grow even when tumor specific $\mathrm{T}$ cells were introduced in the circulation $(29,33)$. The reason for this may be due to the fact that their entry into the tumor masses was limited, or their functions were weakened and defected due to the downregulation of the specific target of antigen or major histocompatibility complex molecules inside the tumor (34). However, it has been demonstrated that, when combined with bacterial infection, the ability of the immune system to kill tumor cells was restored, which may aid in the goal to overcome immune escape mechanisms (28). Combined with Salmonella infection, tumor cells were destroyed by the immune system. In addition, it was demonstrated that tissue debris was captured and taken up by endogenous antigen-presenting cells, and presented on the cell surface of naïve $\mathrm{T}$ cells in order to generate tumor-specific T cells (35). Therefore, investigating the modified Salmonella strain in immunocompetent mice is an important experiment in moving towards clinical trials.

In the present study, an immunocompetent mouse model (BALB/c mice) was used, in which the clearance speed of YB1 in normal tissues was much faster compared with that in nude mice. While nude mice required at least 11 days to completely eliminate YB1, BALB/c required 1-2 days (Fig. 1). This may be due to a more efficient immune system in BALB/c mice compared with nude mice. In light of this improvement, the mice were treated with repeated injections. With high frequency boosting of YB1 every two days for a total of five treatments, the tumor growth was significantly repressed. These results indicated that the involvement of $\mathrm{T}$ cells did enhance the antitumor effects of YB1 treatment. Notably, when BALB/c mice with relatively small tumors $\left(\sim 100 \mathrm{~mm}^{3}\right)$ were treated, a single injection was sufficient to prevent tumor progression (Fig. 3). Using the genetically engineered YB1 strain of Salmonella in cancer therapy demonstrated a promising antitumor effect, as well as high safety (Fig. 4). In conclusion, the approach of engineering Salmonella offers a feasible, effective and safe option for delivering bacteria in cancer therapy. In future studies, live animal imaging (intravital) technology may be applied to investigate the process of bacteria targeting tumors in more detail.

\section{Acknowledgements}

The present study was supported by a grant by the National Science Foundation of China (no. 31200639), awarded to Dr Bin Yu and Dr Lei Shi.

\section{References}

1. Pawelek JM,Low KB and Bermudes D: Bacteria as tumour-targeting vectors. Lancet Oncol 4: 548-556, 2003

2. Yazawa K, Fujimori M, Amano J, Kano Y and Taniguchi S: Bifidobacterium longum as a delivery system for cancer gene therapy: selective localization and growth in hypoxic tumors. Cancer Gene Ther 7: 269-274, 2000.

3. Yazawa K, Fujimori M, Nakamura T: Bifidobacterium longum as a delivery system for gene therapy of chemically induced rat mammary tumors. Breast Cancer Res Treat 66: 165-170, 2001.

4. Taniguchi S, Fujimori M, Sasaki T, Tsutsui H, Shimatani Y, Seki K and Amano J: Targeting solid tumors with non-pathogenic obligate anaerobic bacteria. Cancer Sci 101: 1925-1932, 2010

5. Theys J, Dubois L, Anlezark G, et al: Repeated cycles of Clostridium-directed enzyme prodrug therapy result in sustained antitumour effects in vivo. Br J Cancer 95: 1212-1219, 2006. 
6. Liu SC, Ahn GO, Kioi M, Dorie MJ, Patterson AV and Brown JM Optimized clostridium-directed enzyme prodrug therapy improves the antitumor activity of the novel DNA cross-linking agent PR-104. Cancer Res 68: 7995-8003, 2008.

7. Low KB, Ittensohn M, Le T, et al: Lipid A mutant Salmonella with suppressed virulence and TNFalpha induction retain tumor-targeting in vivo. Nat Biotechnol 17: 37-41, 1999.

8. Zhao M, Yang M, Li XM, et al: Tumor-targeting bacterial therapy with amino acid auxotrophs of GFP-expressing Salmonella typhimurium. Proc Natl Acad Sci USA 102: 755-760, 2005.

9. Zhao M, Yang M, Ma H, et al: Targeted therapy with a Salmonella typhimurium leucine-arginine auxotroph cures orthotopic human breast tumors in nude mice. Cancer Res 66 : 7647-7652, 2006.

10. Yu B Yang M, Shi L, et al: Explicit hypoxia targeting with tumor suppression by creating an 'obligate' anaerobic Salmonella typhimurium strain. Sci Rep 2: 436, 2012.

11. Bertout JA, Patel SA and Simon MC: The impact of $\mathrm{O}_{2}$ availability on human cancer. Nat Rev Cancer 8: 967-975, 2008

12. Hill RP, Marie-Egyptienne DT and Hedley DW: Cancer stem cells, hypoxia and metastasis. Semin Radiat Oncol 19: 106-111, 2009.

13. Hoiseth SK and Stocker BA: Aromatic-dependent Salmonella typhimurium are non-virulent and effective as live vaccines. Nature 291: 238-239, 1981.

14. Animal Research Advisory Committee: Guidelines for Endpoints in Animal Study Proposals. http://oacu.od.nih gov/ARAC/documents/ASP_Endpoints.pdf. Accessed February 1, 2014.

15. Chen G Wei DP, Jia LJ, et al: Oral delivery of tumor-targeting Salmonella exhibits promising therapeutic efficacy and low toxicity. Cancer Sci 100: 2437-2443, 2009.

16. Liao D, Corle C, Seagroves TN and Johnson RS: Hypoxia-inducible factor-1alpha is a key regulator of metastasis in a transgenic model of cancer initiation and progression. Cancer Res 67: 563-572, 2007.

17. Vaupel P: Tumor microenvironmental physiology and its implications for radiation oncology. Semin Radiat Oncol 14: 198-206, 2004.

18. Brown $\mathrm{J}$ and Wilson W: Exploiting tumour hypoxia in cancer treatment. Nat Rev Cancer 4: 437-447, 2004.

19. Movsas B, Chapman JD, Hanlon AL, et al: Hypoxia in human prostate carcinoma: an Eppendorf PO2 study. Am J Clin Oncol 24: 458-461, 2001.
20. Maxwell PH, Pugh CW and Ratcliffe PJ: Activation of the HIF pathway in cancer. Curr Opin Genet Dev 11: 293-299, 2001.

21. Brown JM and Giaccia AJ: The unique physiology of solid tumors: opportunities (and problems) for cancer therapy. Cancer Res 58: 1408-1416,1998.

22. Brown JM and Wilson WR: Exploiting tumour hypoxia in cancer treatment. Nat Rev Cancer 4: 437-447, 2004.

23. Jain RK and Forbes NS: Can engineered bacteria help control cancer? Proc Natl Acad Sci USA 98: 14748-14750, 2001.

24. Chen J Yang B, Cheng X, et al:Salmonella-mediated tumor-targeting TRAIL gene therapy significantly suppresses melanoma growth in mouse model. Cancer Sci 103: 325-333, 2012.

25. Pawelek JM,Low KB and Bermudes D: Tumor-targeted Salmonella as a novel anticancer vector. Cancer Res 57: 4537-4544, 1997.

26. Rosenberg SA Speiss PJ and Kleiner DE: Antitumor effects in mice of the intravenous injection of attenuated Salmonella typhimurium. J Immunother 25: 218-225, 2002.

27. Segre JA, Nemhauser JL, Taylor BA, et al: Positional cloning of the nude locus: genetic, physical, and transcription maps of the region and mutations in the mouse and rat. Genomics 28: 549-559, 1995.

28. Avogadri F, Martinoli C, Petrovska L, et al: Cancer immunotherapy based on killing of Salmonella-infected tumor cells. Cancer Res 65: 3920-3927, 2005.

29. Marincola FM, Wang E, Herlyn M, Seliger B and Ferrone S: Tumors as elusive targets of T-cell-based active immunotherapy. Trends Immunol 24: 335-342, 2003.

30. Cerundolo V, Hermans IF and Salio M: Dendritic cells: a journey from laboratory to clinic. Nat Immunol 5: 7-10, 2004

31. Finn OJ: Cancer vaccines: between the idea and the reality. Nat Rev Immunol 3: 630-641, 2003.

32. Iida N, Dzutsev A, Stewart CA, et al: Commensal bacteria control cancer response to therapy by modulating the tumor microenvironment. Science 342: 967-970, 2013.

33. Lee PP, Yee C, Savage PA, Fong L, Brockstedt D, et al: Characterization of circulating T cells specific for tumor-associated antigens in melanoma patients. Nat Med 5: 677-685, 1999.

34. Garcia-Lora A, Algarra I and Garrido F: MHC class I antigens, immune surveillance, and tumor immune escape. J Cell Physiol 195: 346-355, 2003.

35. Ribas A, Timmerman JM, Butterfield LH and Economou JS: Determinant spreading and tumor responses after peptide-based cancer immunotherapy. Trends Immunol 58-61, 2003. 